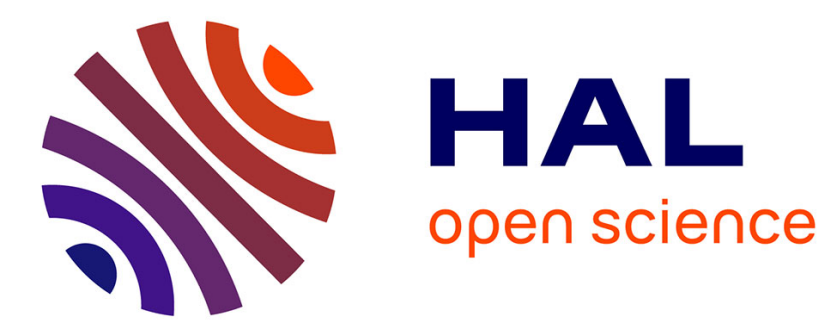

\title{
Fidelity Decay in Trapped Bose-Einstein Condensates
}

Giovanni Manfredi, Paul-Antoine Hervieux

\section{To cite this version:}

Giovanni Manfredi, Paul-Antoine Hervieux. Fidelity Decay in Trapped Bose-Einstein Condensates. Physical Review Letters, 2008, 100 (5), pp.050405. 10.1103/PhysRevLett.100.050405 . hal-00596643

\section{HAL Id: hal-00596643 https://hal.science/hal-00596643}

Submitted on 29 May 2011

HAL is a multi-disciplinary open access archive for the deposit and dissemination of scientific research documents, whether they are published or not. The documents may come from teaching and research institutions in France or abroad, or from public or private research centers.
L'archive ouverte pluridisciplinaire HAL, est destinée au dépôt et à la diffusion de documents scientifiques de niveau recherche, publiés ou non, émanant des établissements d'enseignement et de recherche français ou étrangers, des laboratoires publics ou privés. 


\title{
Fidelity Decay in Trapped Bose-Einstein Condensates
}

\author{
G. Manfredi* and P.-A. Hervieux \\ Institut de Physique et Chimie des Matériaux, CNRS and Université Louis Pasteur, BP 43, F-67034 Strasbourg, France
}

(Received 1 October 2007; published 8 February 2008)

\begin{abstract}
The quantum coherence of a Bose-Einstein condensate is studied using the concept of quantum fidelity (Loschmidt echo). The condensate is confined in an elongated anharmonic trap and subjected to a small random potential such as that created by a laser speckle. Numerical experiments show that the quantum fidelity stays constant until a critical time, after which it drops abruptly over a single trap oscillation period. The critical time depends logarithmically on the number of condensed atoms and on the perturbation amplitude. This behavior may be observable by measuring the interference fringes of two condensates evolving in slightly different potentials.
\end{abstract}

DOI: 10.1103/PhysRevLett.100.050405

PACS numbers: 03.75.Gg, 05.45.Mt

Introduction. - Ultracold atom gases have been at the center of intensive investigations since the first realization of an atomic Bose-Einstein condensate (BEC) in the mid1990s. Applications of BECs include the possibility of revisiting standard problems of condensed-matter physics [1] by making use of periodic optical lattices that mimic the ionic lattice in solid-state systems. More recently, BECs have been used to study quantum transport in disordered systems (another long-standing problem in condensed-matter physics) by using a laser speckle to create a disordered potential. For instance, it was shown that the free expansion of a BEC is restrained, or even completely suppressed, in the presence of a disordered potential [2,3] (see also [4] for a theoretical analysis), an effect akin to Anderson localization in solids [5].

These problems are often approached by studying the interference pattern of two or more BECs that are released from the optical trap, expand freely, and eventually interact with each other [6-9]. Experiments show high-contrast matter-wave interference fringes, thus revealing the coherent nature of Bose-Einstein condensates. Surprisingly, recent experiments have shown high-contrast fringes even for well separated BECs, whose phases are totally uncorrelated [10].

Because the interference pattern depends on the phases of the condensates, the fringes should be sensitive to perturbations that strongly affect the phase, but weakly affect the motion. Therefore, when two condensates are subjected to a random potential (such as that generated by a laser speckle $[11,12]$ ) before interfering, we expect a reduction in the contrast of the interference fringes, which should depend both on the amplitude of the random potential and on the time during which it has been in contact with the condensates. The purpose of this Letter is to propose a theoretical procedure to quantify this loss of coherence and to suggest a possible experimental realization.

In order to estimate the coherence and stability of a quantum system [13], one can compare the evolution of the same initial condition in two slightly different Hamiltonians, $H_{1}=H_{0}+\delta H_{1}$ and $H_{2}=H_{0}+\delta H_{2}$, where $H_{0}$ is the unperturbed Hamiltonian, and $\delta H_{1,2}$ are small perturbations characterized by the same amplitude, same wavelength spectrum, but different phases. The quantum fidelity at time $t$ is then defined as the square of the scalar product of the wave functions evolving with $H_{1}$ and $H_{2}$, respectively: $F(t)=\left|\left\langle\psi_{H_{1}}(t) \mid \psi_{H_{2}}(t)\right\rangle\right|^{2}$. This procedure is sometimes referred to as the "Loschmidt echo," as it is equivalent to evolving the system forward in time with $H_{1}$, then backward with $\mathrm{H}_{2}$, and using the fidelity to check the accuracy of the time reversal.

Virtually all theoretical investigations of the Loschmidt echo consider one-particle systems evolving in a given (usually chaotic) Hamiltonian. Several regimes have been described in the past. For perturbations that are classically weak but quantum-mechanically strong, the fidelity decay is exponential, with a rate independent on the perturbation and given by the classical Lyapunov exponent of the unperturbed system [14]. This behavior has been confirmed by numerical simulations $[15,16]$. For weaker perturbations, the decay rate is still exponential, but perturbation dependent (Fermi golden rule regime). For still weaker perturbations, the decay is Gaussian (perturbative regime) [15]. For integrable systems, other types of decay (notably algebraic) have been observed [17]. Finally, a perturbationindependent regime, though with Gaussian decay, was also observed in experiments [18].

In a previous work [19], we have applied the concept of Loschmidt echo to a system of many electrons interacting through their self-consistent electric field. The numerical results showed that the quantum fidelity remains equal to unity until a critical time, then drops suddenly to much lower values. A similar result was also obtained for a classical system of colliding hard spheres [20]. This effect is probably related to the nonlinearity introduced by the interactions between particles. Therefore, BECs should constitute an ideal arena to determine whether such behavior is typical of many-body quantum systems.

Model. - The dynamics of a BEC is accurately described, in the mean-field approximation, by the GrossPitaevskii equation (GPE). We considered a cigar-shaped 
condensate, where the transverse frequency of the confining potential is much larger then the longitudinal frequency, $\omega_{\perp} \gg \omega_{z}$. In this case, a one-dimensional (1D) approximation can be used, and the GPE reads as:

$$
i \hbar \frac{\partial \psi}{\partial t}=-\frac{\hbar^{2}}{2 m} \frac{\partial^{2} \psi}{\partial z^{2}}+V(z) \psi+g_{1 \mathrm{D}} N_{A}|\psi|^{2} \psi \equiv H_{0} \psi
$$

Here, $\int_{-\infty}^{\infty}|\psi|^{2} d z=1, N_{A}$ is the number of condensed atoms, $g_{1 \mathrm{D}}=2 a \hbar \omega_{\perp}$ is the $1 \mathrm{D}$ effective coupling constant, and $a$ is the 3D scattering length. The confining potential contains a small quartic component, which can be realized optically [21], $V(z)=\frac{1}{2} m \omega_{z}^{2}\left(z^{2}+K z^{4} / L_{\mathrm{ho}}^{2}\right)$, where $L_{\mathrm{ho}}=\left(\hbar / m \omega_{z}\right)^{1 / 2}$ is the harmonic oscillator length.

We choose the parameters of the experiment described in Ref. [2], where $N_{A}=10^{5}$ atoms of ${ }^{87} \mathrm{Rb}(a=5.7 \mathrm{~nm})$ are confined in a quasi-1D trap with $\omega_{z} / 2 \pi=24.7 \mathrm{~Hz}$ and $\omega_{\perp} / 2 \pi=293 \mathrm{~Hz}$. In the simulations, we normalize time to $\omega_{z}^{-1}$, space to $L_{\mathrm{ho}}=2.16 \mu \mathrm{m}$, and energies to $\hbar \omega_{z}$. The dimensionless 1D coupling constant is then $\hat{g}_{1 \mathrm{D}}=$ $g_{1 \mathrm{D}} /\left(L_{\mathrm{ho}} \hbar \omega_{z}\right)=0.063$. The quartic coefficient, which will be crucial to excite a sufficiently complex nonlinear dynamics, is taken to be $K=0.05$. With the above parameters, the half-length of the condensate is roughly $24 \mu \mathrm{m}$.

The random perturbation $\delta H$ can be realized in practice using a laser speckle $[11,12]$. In our simulations, we model the random potential through the sum of a large number of uncorrelated waves: $\delta H / \hbar \omega_{z}=\epsilon \sum_{j=N_{\min }}^{N_{\max }} \cos \left(2 \pi z / \lambda_{j}+\right.$ $\alpha_{j}$ ), where $\epsilon$ is the amplitude of the perturbation, $\lambda_{j}$ 's are the wavelengths, and $\alpha_{j}$ 's are random phases. The smallest wavelength present in the random potential is $\lambda_{\min }=$ $2 L_{\mathrm{ho}}=4.32 \mu \mathrm{m}$, which is consistent with the experimental correlation length, $\sigma_{z}=5 \mu \mathrm{m}$ [2]. The wavelength spectrum of the perturbation (i.e., the values of $N_{\min }$ and $N_{\text {max }}$ ) affects only weakly the behavior of the fidelity; therefore, we will focus our analysis on the dependence of the fidelity on the amplitude $\epsilon$.

In order to compute the quantum fidelity, we proceed as follows: (i) first, we prepare the condensate in its ground state without perturbation; (ii) then, we suddenly displace the anharmonic trap by a distance $\Delta z$ (a few micrometers); (iii) finally, we solve numerically the time-dependent GPE (1) with the perturbed Hamiltonian $H_{0}+\delta H$. Step (iii) is performed for $N=11$ uncorrelated realizations of the random potential, thus yielding $N$ evolutions of the wave function, $\psi_{j}(t)$. We then use all possible combinations to compute the partial fidelities $F_{i j}(t)=\left|\left\langle\psi_{i}(t) \mid \psi_{j}(t)\right\rangle\right|^{2}$. There are, of course, $M=N ! /(N-2) ! 2 !=55$ independent combinations, which are finally averaged to obtain the quantum fidelity, $F(t)=\frac{1}{M} \sum_{j=1}^{M} F_{i j}(t)$. This averaging procedure allowed us to reduce considerably the level of statistical fluctuations.
Results. - Our numerical results showed an unusual behavior for the quantum fidelity, which stays equal to unity until a critical time $\tau_{C}$, and then drops rapidly to small values (Fig. 1). The critical time is defined as the time at which the fidelity has dropped to $60 \%$ of its maximum value, i.e., $F\left(\tau_{C}\right)=0.6$. Interestingly, the fidelity can be nicely fit by a Fermi-like curve

$$
f(t)=\left(1-f_{\infty}\right)\left[1+\exp \left(\frac{t-\tau_{C}}{T}\right)\right]^{-1}+f_{\infty} .
$$

Equation (2) reveals the presence of two distinct time scales: (i) the critical time $\tau_{C}$ and (ii) $T \ll \tau_{C}$, which measures the rapidity of the fidelity decay. The parameter $f_{\infty}$ simply reflects the fact that the fidelity cannot decay to zero, because the system is confined in a finite region in space. In Fig. $1, \omega_{z} T=4.86$ is the same for all three cases and is equal to the oscillation period of a particle trapped in the anharmonic potential $V(z)$, for an initial condition $z(0)=\Delta z, \dot{z}(0)=0$. This means that the fidelity decay occurs over one single oscillation period.

This behavior was confirmed by investigating the dependence of the quantum fidelity on the initial displacement $\Delta z$ (Fig. 2). For each value of $\Delta z$, the parameter $T$ appearing in Eq. (2) is taken to be equal to the corresponding oscillation period in the anharmonic potential $V(z)$. The oscillation period decreases with increasing energy (and thus with increasing $\Delta z$ ) and indeed the fidelity drop becomes steeper for larger displacements. The critical time also slightly increases with decreasing displacement and goes to infinity for $\Delta z \rightarrow 0$. This presumably happens because the dynamics of the condensate becomes too regular when the confinement is harmonic.

Figure 3 shows that $\tau_{C}$ depends logarithmically on the perturbation amplitude, i.e., $\tau_{C} \sim-t_{0} \ln \epsilon$, with $\omega_{z} t_{0} \simeq 3.3$ (this is the straight line depicted in Fig. 3). This is similar to what was obtained for another self-consistent model [19], suggesting that such behavior is generic for $N$-body sys-

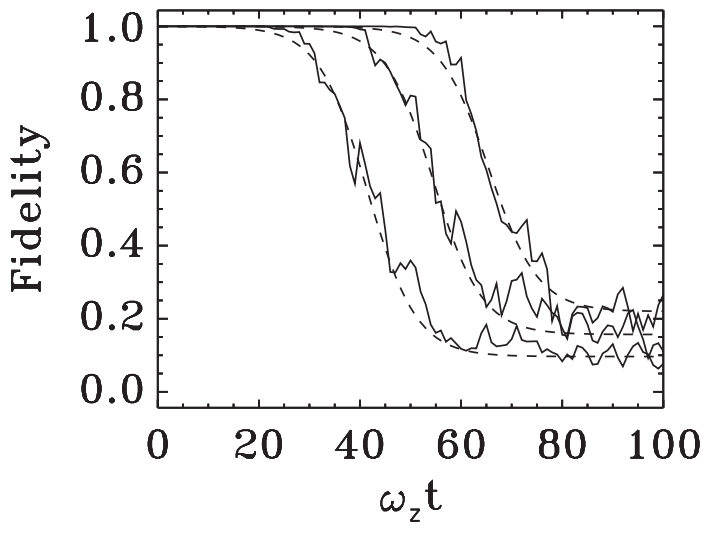

FIG. 1. Fidelity decay for $\Delta z=3 L_{\mathrm{ho}}, N_{A}=10^{5}$, and three values of the perturbation: $\epsilon=10^{-3}, \epsilon=10^{-5}, \epsilon=10^{-7}$ (the critical time increases with decreasing perturbation). The dashed lines are fits obtained using Eq. (2) with $\omega_{z} T=4.86$. 


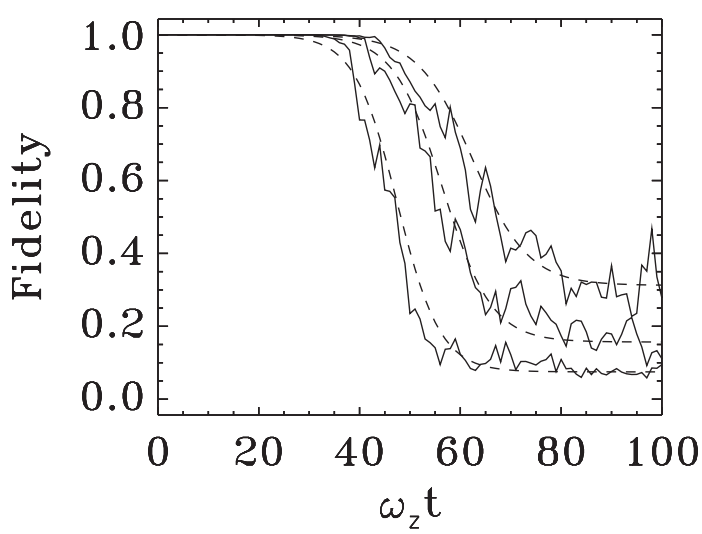

FIG. 2. Fidelity decay for $N_{A}=10^{5}, \epsilon=10^{-5}$, and different values of the displacement $\Delta z=2 L_{\mathrm{ho}}, \Delta z=3 L_{\mathrm{ho}}$, and $\Delta z=$ $4 L_{\mathrm{ho}}$. The steeper curves correspond to larger values of $\Delta z$. The dashed lines are fits obtained using Eq. (2), with $\omega_{z} T=4.27$ $\left(\Delta z=4 L_{\mathrm{ho}}\right), \omega_{z} T=4.86\left(\Delta z=3 L_{\mathrm{ho}}\right)$, and $\omega_{z} T=5.50(\Delta z=$ $\left.2 L_{\mathrm{ho}}\right)$.

tems, at least in the mean-field approximation. The critical time also depends on the number of condensed atoms $N_{A}$. By decreasing $N_{A}, \tau_{C}$ becomes considerably longer (Fig. 4), and for $N_{A} \rightarrow 0$ (i.e., for the linear Schrödinger equation) we have that $\tau_{C} \rightarrow \infty$. For sufficiently large condensates $\left(N_{A} \geq 2 \times 10^{4}\right.$ for the case of Fig. 4), the critical time depends logarithmically on the number of atoms.

Finally, the collapse of the quantum fidelity is clearly linked to the phases of the wave functions. Indeed, by defining an "amplitude fidelity" $F_{a}(t)=\left(\int\left|\psi_{H_{1}} \psi_{H_{2}}\right| d x\right)^{2}$ (which neglects information on the phases), we have verified that $F_{a}(t)$ shows no sign of a sudden collapse when the ordinary fidelity drops.

Discussion. - We have shown that the evolutions of two BECs in slightly different Hamiltonians diverge suddenly

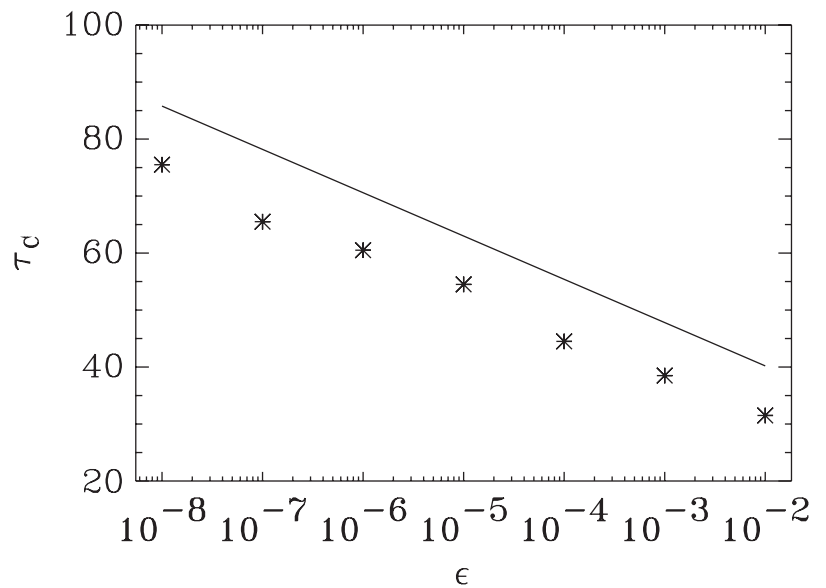

FIG. 3. Critical time $\tau_{C}$ (in units of $\omega_{z}^{-1}$ ) vs perturbation amplitude $\epsilon$, for $\Delta z=3 L_{\mathrm{ho}}$ and $N_{A}=10^{5}$. The solid line represents the curve $\tau_{C} \sim-t_{0} \ln \epsilon$, with $\omega_{z} t_{0}=3.3$. after a critical time $\tau_{C}$. The interaction between the atoms is obviously a vital ingredient, as $\tau_{C} \rightarrow \infty$ when the coupling constant vanishes, i.e., for the linear Schrödinger equation. The crucial point is that, for the GPE, the unperturbed Hamiltonian $H_{0}$ depends on the wave function. When the perturbation induces a small change in $\psi, H_{0}$ is itself modified, which in turn affects $\psi$, and so on. Thanks to such a nonlinear loop, the perturbed and unperturbed solutions can diverge very fast. In contrast, for the singleparticle dynamics $H_{0}$ is fixed, and the solutions only diverge because of the perturbation $\delta H$. Changes in $\psi$ add incrementally to each other, but cannot trigger the nonlinear loop observed in the GP simulations [22].

This behavior is clearly linked to the phases of the wave functions and could be tested experimentally by studying the effect of a random potential on the interference pattern of two condensates [6-9]. A possible experiment could be performed as follows (see Fig. 5). First, a BEC is created in a single-well trap; then, the trap is deformed into a doublewell potential $[9,23]$, with the barrier between the wells sufficiently high that the two condensates cannot tunnel through it. The condensates are left in the double trap for a time long enough to reach their ground state. A laser speckle is then used to create a small random potential of amplitude $\epsilon$ and correlation length $\sigma_{z}$. If $\sigma_{z} \ll d$, where $d$ is the distance between the two BECs, each condensate is subjected to a different random potential with the same statistical properties.

In order to excite the dynamics, the total double-well trap is suddenly shifted by a distance $\Delta z$ of the order of a few micrometers. The BECs evolve in their perturbed trap for a certain time $t$, after which both the trap and the random potential are switched off, so that the condensates can overlap and interfere. We predict that the contrast of the interference fringes will depend on the time $t$ and on the perturbation $\epsilon$, in a manner analogous to the quantum fidelity: if $t<\tau_{C}(\epsilon)$, the contrast should be large, whereas

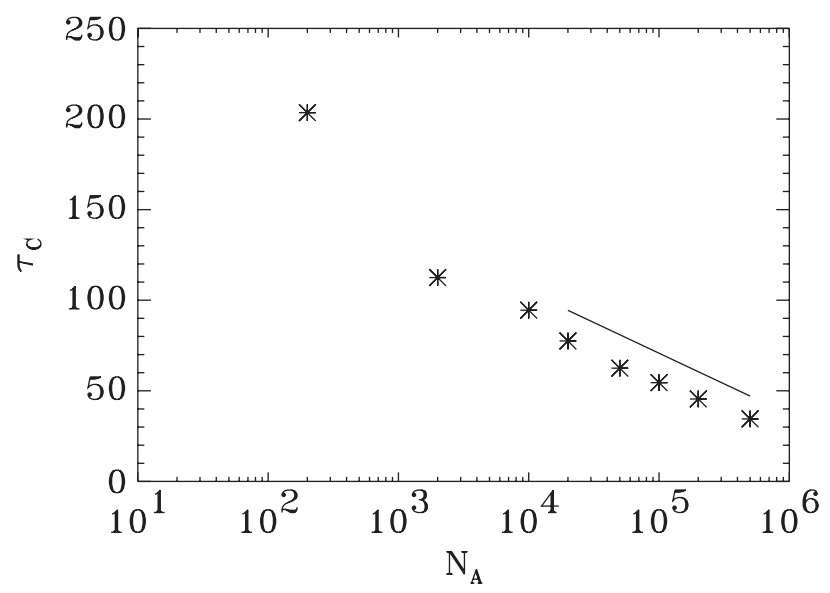

FIG. 4. Critical time $\tau_{C}$ (in units of $\omega_{z}^{-1}$ ) vs number of atoms $N_{A}$ in the condensate, for $\Delta z=3 L_{\mathrm{ho}}$ and $\epsilon=10^{-5}$. The straight line is a guide to the eye. 


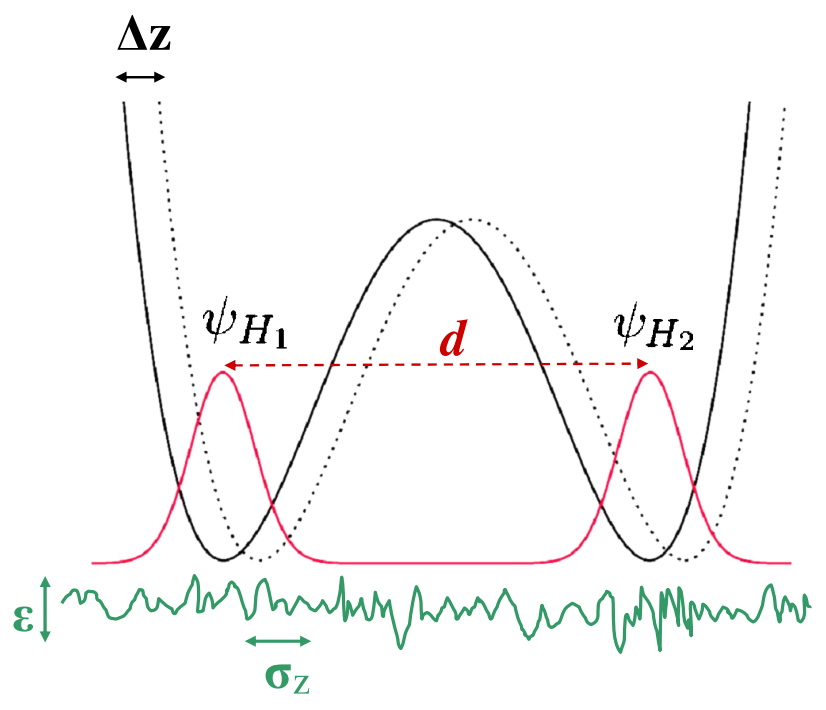

FIG. 5 (color online). Two BECs $\left(\psi_{H_{1}}\right.$ and $\left.\psi_{H_{2}}\right)$ are created in a double-well trap. A random potential with amplitude $\epsilon$ and correlation length $\sigma_{z}$ is superimposed to the confining potential. In order to excite the nonlinear dynamics, the trap is suddenly shifted by a distance $\Delta z$.

it should drop significantly for times larger than $\tau_{C}$. Performing several experiments with different perturbation amplitudes and different numbers of condensed atoms should allow one to reproduce qualitatively the logarithmic scalings of Figs. 3 and 4. Accurate time-resolved measurements might even reproduce the fidelity drop time $T$.

These results, together with those obtained for an electron gas at low temperature [19], suggest that manyparticle systems display a generic sudden decay of the quantum fidelity. Thanks to the ease with which ultracold atom gases can be created and manipulated in the laboratory, BECs should constitute an ideal arena to test these predictions experimentally.

We thank C. Fort for providing the details of the experiment described in Ref. [2]. We also thank R. Jalabert, J. Léonard, and H. Pastawski for several useful suggestions.

*Giovanni.Manfredi@ipcms.u-strasbg.fr

[1] J. R. Anglin and W. Ketterle, Nature (London) 416, 211 (2002).
[2] C. Fort, L. Fallani, V. Guarrera, J. E. Lye, M. Modugno, D. S. Wiersma, and M. Inguscio, Phys. Rev. Lett. 95, 170410 (2005).

[3] D. Clément, A.F. Varon, M. Hugbart, J. A. Retter, P. Bouyer, L. Sanchez-Palencia, D. M. Gangardt, G. V. Shlyapnikov, and A. Aspect, Phys. Rev. Lett. 95, 170409 (2005).

[4] M. Modugno, Phys. Rev. A 73, 013606 (2006).

[5] T. Paul, P. Schlagheck, P. Leboeuf, and N. Pavloff, Phys. Rev. Lett. 98, 210602 (2007).

[6] M. R. Andrews, C. G. Townsend, H.-J. Miesner, D. S. Durfee, D. M. Kurn, and W. Ketterle, Science 275, 637 (1997).

[7] Mark A. Kasevich, Science 298, 1363 (2002).

[8] M. Greiner, I. Bloch, O. Mandel, Th. W. Hänsch, and T. Esslinger, Phys. Rev. Lett. 87, 160405 (2001).

[9] Y. Shin, M. Saba, T. A. Pasquini, W. Ketterle, D.E. Pritchard, and A.E. Leanhardt, Phys. Rev. Lett. 92, 050405 (2004).

[10] Z. Hadzibabic, S. Stock, B. Battelier, V. Bretin, and J. Dalibard, Phys. Rev. Lett. 93, 180403 (2004).

[11] J. E. Lye, L. Fallani, M. Modugno, D. S. Wiersma, C. Fort, and M. Inguscio, Phys. Rev. Lett. 95, 070401 (2005).

[12] D. Clément, A. F. Varon, J. A. Retter, L. Sanchez-Palencia, A. Aspect, and P. Bouyer, New J. Phys. 8, 165 (2006).

[13] A. Peres, Phys. Rev. A 30, 1610 (1984).

[14] R. A. Jalabert and H. M. Pastawski, Phys. Rev. Lett. 86, 2490 (2001).

[15] Ph. Jacquod, P. G. Silvestrov, and C. W. J. Beenakker, Phys. Rev. E 64, 055203(R) (2001).

[16] F. M. Cucchietti, C. H. Lewenkopf, E. R. Mucciolo, H. M. Pastawski, and R. O. Vallejos, Phys. Rev. E 65, 046209 (2002).

[17] G. Benenti, G. Casati, and G. Veble, Phys. Rev. E 68, 036212 (2003).

[18] H. M. Pastawski, P. R. Levstein, G. Usaj, J. Raya, and J. Hirschinger, Physica (Amsterdam) 283A, 166 (2000).

[19] G. Manfredi and P.-A. Hervieux, Phys. Rev. Lett. 97, 190404 (2006).

[20] R. Pinto, E. Medina, and H. M. Pastawski (private communication).

[21] V. Bretin, S. Stock, Y. Seurin, and J. Dalibard, Phys. Rev. Lett. 92, 050403 (2004).

[22] As the GPE is intrinsically nonlinear, one could, in principle, be able to merely perturb the initial quantum state (without doing anything to the Hamiltonian) in order to see some nontrivial decay of the corresponding overlap function. It will be interesting to investigate this alternative approach in future studies.

[23] W. Hänsel, J. Reichel, P. Hommelhoff, and T. W. Hänsch, Phys. Rev. A 64, 063607 (2001). 